\title{
Antimicrobial polypeptides in host defense of the respiratory tract
}

\author{
Tomas Ganz \\ University of California-Los Angeles School of Medicine, Center for the Health Sciences, \\ 10833 LeConte Avenue, 37-055, Los Angeles, California 90095-1690, USA. \\ Phone: (310) 825-6112; Fax: (310) 206-8766; E-mail: tganz@ucla.edu. \\ J. Clin. Invest. 109:693-697 (2002). DOI:10.1172/JCI200215218.
}

The large surfaces of the respiratory tract are often the initial site of contact between microbes and their human hosts. Respiratory epithelia are coated with a thin layer of airway and alveolar secretions whose composition varies by anatomical location and in response to mechanical, chemical, and microbial stimulation. In the nose, trachea, and bronchi, the secretions are generated by airway epithelial cells, especially the goblet cells; by the submucosal glands; by transudation and transport of proteins from plasma; and by resident and recruited phagocytes - neutrophils, eosinophils, monocytes, and macrophages. In the distal airways and alveoli, Clara cells and type 2 alveolar cells are, respectively, the predominant secretory epithelial cells. As first described by Alexander Fleming nearly 80 years ago (1), respiratory secretions have microbicidal and microbistatic properties mediated by their constituent antimicrobial polypeptides. These intrinsic antimicrobial properties of respiratory secretions act in concert with the mechanical and phagocytic clearance mechanisms, described elsewhere in this Perspective series, to defend the respiratory tract against colonization or invasion by environmental microbes.

\section{Antimicrobial activity of respiratory secretions}

Several recent studies have confirmed and greatly extended Fleming's original observations on the antimicrobial properties of respiratory secretions collected from human donors. Travis et al. (2) found that respiratory secretions harvested by nasal or bronchoalveolar lavage, or as cystic fibrosis sputum, showed antimicrobial activity against a test strain of Escherichia coli, with a clear dependence on the ionic strength of the medium. Under low ionic strength conditions, both nasal and lung secretions were antimicrobial, as were their cationic polypeptide constituents - lysozyme, lactoferrin, secretory leukoprotease inhibitor (SLPI), and neutrophil and epithelial defensins. However, the antimicrobial activity of lavage fluids and their polypeptide constituents could be inhibited by increasing ionic strength and was especially sensitive to divalent cations. These findings were consistent with the well-established ability of ionic constituents to inhibit the electrostatic attraction of cationic polypeptides to anionic microbial surfaces. In addition, binding of divalent cations to outer membranes of Gram-negative bacteria is known to stabilize the outer membrane against penetration by cationic polypeptides. Mucin, an abundant polyanionic component of respiratory secretions, and small, uncharged solutes that increase osmolarity but not ionic strength, had no effect on the antimicrobial activity of lavage fluids, lysozyme, or lactoferrin.

Cole et al. (3) in our group employed a complementary approach, examining minimally manipulated nasal fluid collected with a suction catheter that stimulated the nasal mucosa of normal donors. In this study, the ionic concentrations or the concentrations of the polypeptide constituents of nasal fluid were maintained in their natural state. Nasal fluid from most donors killed or inhibited the growth of Staphylococcus aureus, E. coli, and Psendomonas aeruginosa, but fluids from nasal carriers of $S$. aureus permitted the growth of this bacterium. Moreover, at least in some experiments, $P$. aeruginosa recovered its ability to grow after 24 hours of incubation, indicating that surviving bacteria could adapt to the injurious effects of nasal fluid.

The inhibition or killing of bacteria by nasal fluid is not due to its intrinsic inability to provide nutrients for bacterial growth, since brief heat treatment of the fluid at $100^{\circ} \mathrm{C}$ renders it permissive for the growth of a cystic fibrosis isolate of $P$. aeruginosa. Nasal secretions from which cationic polypeptides are removed by treatment with cation exchangers likewise become permissive for the growth of $P$. aeruginosa, and the activity can be restored by adding back the cationic fraction (A. Cole and T. Ganz, unpublished observations). Yet another study, this one testing the effects of bronchoalveolar lavage fluid (BALF) on the sensitive test bacterium Bacillus subtilis, identified a series of antimicrobial polypeptides - lysozyme, neutrophil defensins, SLPI, and the cathelicidin LL-37 among them - in both sarcoidosis patients and normal donors (4).

These reports confirm the concept that respiratory secretions are broadly antimicrobial and that their activity is largely due to their cationic polypeptide components. They also indicate that the antimicrobial 
activity of respiratory fluid is rather precarious, in that it can be abolished by altering the ionic milieu, by bacterial adaptation, and by as-yet uncharacterized abnormalities in nasal carriers of $S$. aureus.

Studies of human airway epithelia maintained either in vitro or in nude mice demonstrate that respiratory epithelial cells generate antimicrobial activity even in the absence of glandular structures $(5,6)$. However, it has become clear that, compared with respiratory secretions collected in vivo, the antimicrobial activity of cultured airway epithelia is much less potent, perhaps because these cell culture models secrete a low-protein fluid that contains scant amounts of the major substances found in airway secretions (lysozyme, lactoferrin, SLPI, and defensins). In murine models, differentiation of airway epithelia into mucus-producing cells is dependent on Th2 cytokines IL-4, $-5,-9$, and -13 , which may act predominantly through IL-9 (7). It remains to be shown whether the high-output production of antimicrobial proteins is also dependent on cytokineinduced differentiation of airway epithelial cells.

\section{The composition of respiratory secretions}

Since the epithelial fluid layer is very thin under resting conditions, the collection of sufficient volume of respiratory fluid from live human or animal donors either subjects the epithelium to mechanical or chemical stimulation or depends on pathologic processes that increase and unavoidably modify secretions. Moreover, the commonly used bronchoalveolar lavage method admixes secretions from anatomically diverse locations and dilutes them to an unknown extent. Because of these obstacles, the ionic and polypeptide composition of resting respiratory fluid in specific anatomical locations is subject to substantial uncertainty. However, mixed samples of respiratory fluid obtained from the nose by mechanical or chemical stimulation $(3,8)$, or as sputum from patients with chronic bronchitis (9-11), indicate that lysozyme and lactoferrin are the most abundant antimicrobial proteins of airway secretions, at about $0.1-1 \mathrm{mg} / \mathrm{ml}$. These estimates agree with bronchoalveolar lavage sampling $(2,12)$, assuming that the BALF represents an approximately hundredfold dilution of respiratory fluid, an approximate (and controversial) dilution factor based on measurements of urea concentrations in BALF (13). SLPI is about tenfold less abundant $(14,15)$.

The concentrations of neutrophil and epithelial defensins in respiratory fluid change dramatically with acute inflammation. In cystic fibrosis sputum, the concentrations of neutrophil defensins range from $300 \mu \mathrm{g}$ to more than $1 \mathrm{mg} / \mathrm{ml}$ (16), but concentrations in BALF of patients undergoing bronchoscopy without clinical signs of inflammation are only about $100 \mathrm{ng} / \mathrm{ml}$ (17), corresponding to about $10 \mu \mathrm{g} / \mathrm{ml}$ in respiratory fluid. Concentrations of epithelial human $\beta$-defensins HBD-1 and HBD-2 in inflamed nasal fluid and BALF are about a thousandfold lower than those of lysozyme, i.e., about $1 \mu \mathrm{g} / \mathrm{ml}(3,18)$. Expression of antimicrobial polypeptides is modulated locally by inflammation. Inflammatory stimuli release chemoattractants that recruit neutrophils containing large amounts of lysozyme, lactoferrin, and neutrophil defensins. These stimuli also increase the synthesis of $\beta$-defensin by epithelial cells $(18$, 19) and, when chronic, induce the differentiation of respiratory epithelial cells into secretory cell types.

\section{Activities of antimicrobial polypeptides from respiratory secretions}

Lysozyme is a $14-\mathrm{kDa}$ enzyme directed against the $\beta$ $1 \rightarrow 4$ glycosidic bond between $N$-acetylglucosamine and $N$-acetylmuramic acid residues that make up peptidoglycan, the cell wall material that gives bacteria their shape. In addition to enzymatic lysis of bacterial cell walls, lysozyme can also kill bacteria by a nonenzymatic mechanism (20). Lysozyme is highly active against many Gram-positive species, including Bacillus megaterium, Micrococcus luteus, and many streptococci, but it appears to be ineffective against Gram-negative bacteria (21) unless potentiated by certain cofactors (lactoferrin, antibody-complement complexes, or hydrogen peroxide and ascorbic acid). These cofactors presumably disrupt the outer membrane of Gram-negative bacteria and allow lysozyme access to the sensitive peptidoglycan layer.

Lysozyme is a component of both phagocytic and secretory granules of neutrophils and is also produced by monocytes, macrophages, and epithelial cells. It is about tenfold more abundant in the initial "airway" aliquot than in subsequent aliquots of bronchoalveolar lavage (12), and its concentration correlates poorly with neutrophil concentrations, suggesting that, on the average, airway epithelium and its glands are the major sources of lysozyme in airway secretions. Mice overexpressing lysozyme under the control of the human Surfactant protein $C$ promoter show increased resistance to lung infection with group B Streptococcus or P. aeruginosa (22).

Lactoferrin, a close relative of the serum protein transferrin, is an $80-\mathrm{kDa}$ iron-binding protein highly abundant in the specific granules of human neutrophils and in epithelial secretions. Lactoferrin inhibits microbial growth by sequestering iron essential for microbial respiration (23). It can also be directly microbicidal (24), an activity that is concentrated in its N-terminal cationic fragment "lactoferricin."

SLPI is a $12-\mathrm{kDa}$ nonglycosylated protein consisting of two similar domains. The $\mathrm{N}$-terminal domain has modest antimicrobial activity in vitro against both Gram-negative and Gram-positive bacteria (25). The C-terminal domain acts as an effective inhibitor of neutrophil elastase and may also be involved in intracellular regulation of responses to LPS (26).

Human defensins (27) are 3- to 5-kDa peptides, members of a widely distributed family of microbicidal peptides with a characteristic three-dimensional fold and sixcysteine/three-disulfide pattern. Although the human genome project indicates that the family contains more than 20 potentially expressed genes, relatively few human defensin peptides have been characterized to date. Three closely related defensins, human neutrophil peptides HNP-1, -2 , and -3 , are major components of the dense azurophil granules of neutrophils, and a fourth, HNP-4, is found in the same location but is much less abundant. Two other human defensins $(28,29)$, HD-5 and -6 , are 
located in the lysozyme-rich secretory granules of Paneth cells, an epithelial cell type positioned at the bottom of small intestinal crypts, and are thought to be involved in local host defense. The three most recently characterized defensins, HBD-1 (30, 31), HBD-2 (32), and HBD-3 (33, $34)$, differ slightly from the classical $\alpha$-defensins in the spacing and connectivity of their cysteines. Their mRNAs are expressed in epithelia, with HBD-1 being most abundant in the kidney, and HBD-2 and -3 in inflamed skin, but all three are detectable in the respiratory tract as well $(18,33,35,36)$. As with the bovine tracheal antimicrobial peptide (37), the prototypic airway defensin, the synthesis and secretion of HBD-2 (and presumably HBD-3) are regulated in a dual fashion, first by direct epithelial responses to LPS and other microbial stimuli and second by cytokines. The former, higher-threshold effect is most likely mediated by epithelial CD14, Toll-like receptors, and the transcription factor NF-KB (19). The lower-threshold, cytokine-mediated response is triggered primarily by the encounter of microbes with local macrophages. These cells then produce IL- $1 \alpha$ and IL- $1 \beta$ and other cytokines (18), which in turn act on epithelial cytokine receptors to increase epithelial defensin synthesis. This scheme avoids promiscuous activation by low concentrations of inhaled noninvasive microbes while retaining the ability to activate in response to large boluses of microbes or epithelial penetration by fewer invasive microbes.

In vitro, under low-salt conditions (e.g., $10 \mathrm{mM}$ sodium phosphate), defensins are microbicidal at micromo$\operatorname{lar}(\sim \mu \mathrm{g} / \mathrm{ml})$ concentrations against many Gram-positive and Gram-negative bacteria, yeast and fungi, and certain enveloped viruses. Increasing salt concentrations competitively inhibit defensin activity, with the inhibitory effect modulated by the properties of the target microbe. Defensins act preferentially on microbes by permeabilizing microbial membranes rich in anionic phospholipids, with relative sparing of the cholesterol- and neutral phospholipid-rich cell membranes of the host.

Estimates of defensin concentrations in the phagocytic vacuoles of neutrophils are in the milligrams-per-milliliter range, a concentration that should be sufficient to overcome inhibition by extracellular ion concentrations (see Knowles and Boucher, this Perspective series, ref. 38). Similar considerations also apply to the activity of defensins in the narrow intestinal crypts $(5-10 \mu \mathrm{m}$ in diameter) into which Paneth cells secrete their defensincontaining granules (39). The concentration of HBD-2 in desquamated inflamed skin is in the range of $10 \mu \mathrm{g} / \mathrm{ml}$ (32), again sufficient to inhibit or kill many microbes. In the respiratory tract, the concentrations of neutrophil defensins HNP-1 through -4 and epithelial HBD-2 are greatly increased by inflammation $(16,18)$ and could reach antimicrobial concentrations, but their specific role in antimicrobial events in the lung has not yet been documented. It should be noted that some defensins manifest additional in vitro activities that may contribute to inflammation and repair, including inhibition of adrenocorticotropic hormone-stimulated cortisol production, inhibition of fibrinolysis, mitogenic effects on fibroblasts (40), and induction of IL-8 and SLPI secretion by bronchial epithelial cells $(41,42)$. Their recently described chemoattractant effects on immature dendritic cells and memory T cells $(43,44)$ are likely to be important in linking the initial innate response to infection to the later development of acquired immunity.

Cathelicidins (45) are members of a large family of mammalian microbicidal peptides with a conserved $\mathrm{N}$ terminal precursor structure (containing typically about 100 amino acid residues) but highly heterogeneous C-terminal peptides (10-40 amino acid residues). Most cathelicidins undergo extracellular proteolytic cleavage that frees the $\mathrm{C}$-terminal peptide from the precursor and activates it (46). The sole known human cathelicidin has been named hCAP18 and FALL-39/LL-37 by the three groups that described its cDNA, gene, and peptide forms (47-49). Its abundance in neutrophil-specific granules appears to be about a third of that of lactoferrin or lysozyme, the two major proteins of specific granules. Exemplifying the overlap of phagocytic and epithelial host defenses, the mRNA for the human cathelicidin is also found in the testes, in inflamed human keratinocytes (50), in other squamous epithelia (51), and in airway epithelia (52). In vitro, the human peptide LL-37 displays both LPS-binding (47) and broad-spectrum microbicidal activities $(52,53)$. Overexpression of LL-37 in an airway xenograft model of cystic fibrosis restores the deficient antimicrobial activity (6), indicating that LL-37 retains activity in the complex milieu of the airway.

These various antimicrobial proteins and peptides differ in their molecular specificity, targeting phospholipid membranes, peptidoglycans, or iron. They also vary in their mode of action on that target, acting as hydrolytic enzymes or creating pores in the bacterial cell wall, for example. They might therefore be expected to act cooperatively by attacking multiple essential structures in bacteria or by unmasking structures vulnerable to other components of the mixture. The multiplicity of active ingredients may also broaden the effectiveness of the mixture against the many potential microbial targets and decrease the likelihood of acquired resistance. Indeed, Travis et al. (2) have recently provided experimental evidence for synergistic interactions between lysozyme, lactoferrin, and SLPI, the main polypeptide constituents of airway secretions.

\section{Interventions to enhance antimicrobial polypeptide-mediated host defense mechanisms}

The selection of antimicrobial peptides and proteins as therapeutic candidates is governed by considerations of biological activity, toxicity, and the costs of developing, producing, and administering these compounds. Since the antimicrobial activities of these substances usually require at least micromolar concentrations, and the costs of production rise with the size of the polypeptide and its structural complexity, initial efforts have centered on relatively small peptides, or small fragments of larger proteins. The costs and toxicities increase with the volume of distribution in the body, favoring topical over systemic administration for initial trials of these new compounds. Antibiotics based on small peptides of human or animal origin might also be predicted to elicit fewer allergic reactions than many conventional antibiotics of bacterial or fungal origin. For pulmonary applications, use of 
antimicrobial peptides as inhaled antibiotics is particularly attractive. Among conventional antibiotics, only tobramycin is currently approved for this application, and microbial resistance to this agent is on the rise. The pathogenic role of $P$. aeruginosa and similar bacteria in cystic fibrosis presents a unique opportunity to test the pharmacologic use of antimicrobial peptides that are variants of naturally occurring forms. Initial clinical trials of a protegrin (porcine neutrophil cathelicidin) derivative (54) for this and other topical applications are ongoing.

Alternative strategies, at an earlier stage of development, include the use of gene therapy technology to deliver functional antimicrobial peptide genes to the respiratory epithelium (6) or systemically (55).

\section{Summary and future prospects}

The increasing interest in mechanisms of innate immunity has refocused attention on the highly effective processes that keep the lower respiratory tract largely free of microbes. The picture emerging is far from the passive system depicted in a rudimentary form in most current immunology texts. Antimicrobial polypeptides, a part of the innate host defense system, are present constitutively and in high concentrations on respiratory epithelia but are also locally supplemented by secretion from recruited phagocytes or from activated epithelial cells. They selectively target vital microbial structures, taking advantage of structural and biochemical differences between the host and the microbes. Some antimicrobial polypeptides also function as signaling molecules, alerting the adaptive immune system to the presence of invaders. Several diseases characterized by persistent mucosal colonization or infection, including cystic fibrosis (5) and nasal colonization with $S$. aureus, may turn out to be manifestations of defects on antimicrobial polypeptide-based defenses. For these and other airway infections, the use of inhaled or topical synthetic peptide antimicrobials is an exciting prospect.

Areas of ongoing scientific ferment are likely to include highly selective microsampling and analysis of the composition of respiratory secretions in specific locations in the respiratory system, including alveoli, small airways, and the bronchi. Continued attention will be paid to the possible role of antimicrobial polypeptides and other innate host defense components in the early pathogenesis of cystic fibrosis in the lung. Much will be learned about antimicrobial polypeptides by knocking out genes or clusters of genes that encode antimicrobial polypeptides, especially when this becomes possible in larger animals whose respiratory systems are similar to those of humans. An alternative strategy to define the specific function of antimicrobial polypeptides, selective pharmaceutical inhibition of their posttranslational proteolytic activation, may be productive in animals where the knockout technology is not yet available (56). Finally, we can also look forward to improved antimicrobial therapies that take advantage of potential synergies with the "natural antibiotics" already present at the site of infection.

\section{Acknowledgments}

My laboratory has received support from the NIH, The Will Rogers Fund, and the Cystic Fibrosis Foundation. I would like to thank Robert Lehrer, Charles Bevins, Alexander Cole, and John Fiddes for discussions that stimulated some of the ideas in this manuscript. I apologize to those colleagues whose primary work could not be cited because of space limitations. During the preparation of this manuscript, I was a member of the scientific advisory board of Intrabiotics Pharmaceuticals Inc., a company engaged in the commercial development of antimicrobial peptides.

1. Fleming, A. 1922. On a remarkable bacteriolytic element found in tissues and secretions. Proc. R. Soc. Lond. B Biol. Sci. 93:306-317.

2. Travis, S.M., et al. 1999. Activity of abundant antimicrobials of the human airway. Am. J. Respir. Cell Mol. Biol. 20:872-879.

3. Cole, A.M., Dewan, P., and Ganz, T. 1999. Innate antimicrobial activity of nasal secretions. Infect. Immun. 67:3267-3275.

4. Agerberth, B., et al. 1999. Antibacterial components in bronchoalveolar lavage fluid from healthy individuals and sarcoidosis patients. Am.J. Respir. Crit. Care Med. 160:283-290.

5. Smith, J.J., Travis, S.M., Greenberg, E.P., and Welsh, M.J. 1996. Cystic fibrosis airway epithelia fail to kill bacteria because of abnormal airway surface fluid. Cell. 85:229-236.

6. Bals, R., Weiner, D.J., Meegalla, R.L., and Wilson, J.M. 1999. Transfer of a cathelicidin peptide antibiotic gene restores bacterial killing in a cystic fibrosis xenograft model. J. Clin. Invest. 103:1113-1117.

7. Wills-Karp, M. 2000. Trophic slime, allergic slime. Am. J. Respir. Cell Mol. Biol. 22:637-639.

8. Raphael, G.D., et al. 1989. Pathophysiology of rhinitis. Lactoferrin and lysozyme in nasal secretions. J. Clin. Invest. 84:1528-1535.

9. Brogan, T.D., Ryley, H.C., Neale, L., and Yassa, J. 1975. Soluble proteins of bronchopulmonary secretions from patients with cystic fibrosis, asthma, and bronchitis. Thorax. 30:72-79.

10. Harbitz, O., Jenssen, A.O., and Smidsrod, O. 1984. Lysozyme and lactoferrin in sputum from patients with chronic obstructive lung disease. Eur. J. Respir. Dis. 65:512-520.

11. Kotlar, H.K., Harbitz, O., Jenssen, A.O., and Smidsrod, O. 1980. Quantitation of proteins in sputum from patients with chronic obstructive lung disease. II. Determination of albumin, transferrin, alpha1-acid glycoprotein, IgG, IgM, lysozyme and C3-complement factor. Eur. J. Respir. Dis. 61:233-239.

12. Thompson, A.B., Bohling, T., Payvandi, F., and Rennard, S.I. 1990. Lower respiratory tract lactoferrin and lysozyme arise primarily in the airways and are elevated in association with chronic bronchitis. J. Lab. Clin. Med. 115:148-158.

13. Rennard, S.I., et al. 1986. Estimation of volume of epithelial lining fluid recovered by lavage using urea as marker of dilution. J. Appl. Physiol. 60:532-538.

14. Hill, A.T., Bayley, D.L., Campbell, E.J., Hill, S.L., and Stockley, R.A. 2000. Airways inflammation in chronic bronchitis: the effects of smoking and alpha1-antitrypsin deficiency. Eur. Respir. J. 15:886-890.

15. Lee, C.H., et al. 1993. Distribution of secretory leukoprotease inhibitor in the human nasal airway. Am. Rev. Respir. Dis. 147:710-716.

16. Soong, L.B., Ganz, T., Ellison, A., and Caughey, G.H. 1997. Purification and characterization of defensins from cystic fibrosis sputum. Inflamm. Res. 46:98-102.

17. Schnapp, D., and Harris, A. 1998. Antibacterial peptides in bronchoalveolar lavage fluid. Am. J. Respir. Cell Mol. Biol. 19:352-356.

18. Singh, P.K., et al. 1998. Production of $\beta$-defensins by human airway epithelia. Proc. Natl. Acad. Sci. USA. 95:14961-14966.

19. Becker, M.N., Diamond, G., Verghese, M.W., and Randell, S.H. 2000. CD14-dependent lipopolysaccharide-induced beta-defensin-2 expression in human tracheobronchial epithelium. J. Biol. Chem. 275:29731-29736.

20. Laible, N.J., and Germaine, G.R. 1985. Bactericidal activity of human lysozyme, muramidase-inactive lysozyme, and cationic polypeptides against Streptococcus sanguis and Streptococcus faecalis: inhibition by chitin oligosaccharides. Infect. Immun. 48:720-728.

21. Ellison, R.T., and Giehl, T.J. 1991. Killing of gram-negative bacteria by lactoferrin and lysozyme. J. Clin. Invest. 88:1080-1091.

22. Akinbi, H.T., Epaud, R., Bhatt, H., and Weaver, T.E. 2000. Bacterial killing is enhanced by expression of lysozyme in the lungs of transgenic mice. J. Immunol. 165:5760-5766.

23. Arnold, R.R., Cole, M.F., and McGhee, J.R. 1977. A bactericidal effect for human lactoferrin. Science. 197:263-265.

24. Arnold, R.R., Russell, J.E., Champion, W.J., Brewer, M., and Gauthier, J.J. 
1982. Bactericidal activity of human lactoferrin: differentiation from the stasis of iron deprivation. Infect. Immun. 35:792-799.

25. Hiemstra, P.S., et al. 1996. Antibacterial activity of antileukoprotease. Infect. Immun. 64:4520-4524.

26. Zhu, J., Nathan, C., and Ding, A. 1999. Suppression of macrophage responses to bacterial lipopolysaccharide by a non-secretory form of secretory leukocyte protease inhibitor. Biochim. Biophys. Acta. 1451:219-223.

27. Lehrer, R.I., Lichtenstein, A.K., and Ganz, T. 1993. Defensins: antimicrobial and cytotoxic peptides of mammalian cells. Annu. Rev. Immunol. 11:105-128.

28. Jones, D.E., and Bevins, C.L. 1992. Paneth cells of the human small intestine express an antimicrobial peptide gene. J. Biol. Chem. 267:23216-23225.

29. Jones, D.E., and Bevins, C.L. 1993. Defensin- 6 mRNA in human Paneth cells: implications for antimicrobial peptides in host defense of the human bowel. FEBS Lett. 315:187-192.

30. Bensch, K.W., Raida, M., Magert, H.J., Schulz-Knappe, P., and Forssmann, W.G. 1995. hBD-1: a novel beta-defensin from human plasma. FEBS Lett. 368:331-335.

31. Valore, E.V., et al. 1998. Human beta-defensin-1: an antimicrobial peptide of urogenital tissues. J. Clin. Invest. 101:1633-1642.

32. Harder, J., Bartels, J., Christophers, E., and Schroeder, J.-M. 1997. A peptide antibiotic from human skin. Nature. 387:861-862.

33. Harder, J., Bartels, J., Christophers, E., and Schroder, J.M. 2001. Isolation and characterization of human beta-defensin-3, a novel human inducible peptide antibiotic. J. Biol. Chem. 276:5707-5713.

34. Jia, H.P., et al. 2001. Discovery of new human beta-defensins using a genomics-based approach. Gene. 263:211-218.

35. Zhao, C.Q., Wang, I., and Lehrer, R.I. 1996. Widespread expression of beta-defensin HBD-1 in human secretory glands and epithelial cells. FEBS Lett. 396:319-322.

36. Bals, R., et al. 1998. Human beta-defensin 2 is a salt-sensitive peptide antibiotic expressed in human lung. J. Clin. Invest. 102:874-880.

37. Diamond, G., Russell, J.P., and Bevins, C.L. 1996. Inducible expression of an antibiotic peptide gene in lipopolysaccharide-challenged tracheal epithelial cells. Proc. Natl. Acad. Sci. USA. 93:5156-5160.

38. Knowles, M.R., and Boucher, R.C. 2002. Mucus clearance as a primary innate defense mechanism for mammalian airways. J. Clin. Invest. 109:571-577. DOI:10.1172/JCI200215217.

39. Ayabe, T., et al. 2000. Secretion of microbicidal $\alpha$-defensins by intestinal Paneth cells in response to bacteria. Nat. Immun. 1:113-118.

40. Ganz, T., and Lehrer, R.I. 1995. Defensins. Pharmacol. Ther. 66:191-205.
41. Van Wetering, S., et al. 1997. Effect of defensins on interleukin-8 synthesis in airway epithelial cells. Am. J. Physiol. 272:L888-L896.

42. Van Wetering, S., et al. 2000. Regulation of SLPI and elafin release from bronchial epithelial cells by neutrophil defensins. Am. J. Physiol. Lung Cell. Mol. Physiol. 278:L51-L58.

43. Yang, D., et al. 1999. Beta-defensins: linking innate and adaptive immunity through dendritic and T cell CCR6. Science. 286:525-528.

44. Ganz, T. 1999. Defensins and host defense. Science. 286:420-421.

45. Zanetti, M., Gennaro, R., and Romeo, D. 1995. Cathelicidins: a novel protein family with a common proregion and a variable C-terminal antimicrobial domain. FEBS Lett. 374:1-5.

46. Sorensen, O.E., et al. 2001. Human cathelicidin, hCAP-18, is processed to the antimicrobial peptide LL-37 by extracellular cleavage with proteinase 3. Blood. 97:3951-3959.

47. Larrick, J.W., et al. 1995. Human CAP18: a novel antimicrobial lipopolysaccharide-binding protein. Infect. Immun. 63:1291-1297.

48. Gudmundsson, G.H., et al. 1996. The human gene FALL39 and processing of the cathelin precursor to the antibacterial peptide LL-37 in granulocytes. Eur. J. Biochem. 238:325-332.

49. Sorensen, O., Arnljots, K., Cowland, J.B., Bainton, D.F., and Borregaard, N. 1997. The human antibacterial cathelicidin, hCAP-18, is synthesized in myelocytes and metamyelocytes and localized to specific granules in neutrophils. Blood. 90:2796-2803.

50. Frohm, M., et al. 1997. The expression of the gene coding for the antibacterial peptide LL-37 is induced in human keratinocytes during inflammatory disorders. J. Biol. Chem. 272:15258-15263.

51. Frohm, N.M., et al. 1999. The human cationic antimicrobial protein (hCAP18), a peptide antibiotic, is widely expressed in human squamous epithelia and colocalizes with interleukin-6. Infect. Immun. 67:2561-2566.

52. Bals, R., Wang, X., Zasloff, M., and Wilson, J.M. 1998. The peptide antibiotic LL-37/hCAP-18 is expressed in epithelia of the human lung where it has broad antimicrobial activity at the airway surface. Proc. Natl. Acad. Sci. USA. 95:9541-9546.

53. Turner, J., Cho, Y., Dinh, N.N., Waring, A.J., and Lehrer, R.I. 1998. Activities of LL-37, a cathelin-associated antimicrobial peptide of human neutrophils. Antimicrob. Agents Chemother. 42:2206-2214.

54. Ganz, T., Bellm, L., and Lehrer, R.I. 2000. Protegrins: new antibiotics of mammalian origin. Expert Opin. Investig. Drugs. 9:1731-1742.

55. Bals, R., Weiner, D.J., Moscioni, A.D., Meegalla, R.L., and Wilson, J.M. 1999. Augmentation of innate host defense by expression of a cathelicidin antimicrobial peptide. Infect. Immun. 67:6084-6089.

56. Cole, A.M., et al. 2001. Inhibition of neutrophil elastase prevents cathelicidin activation and impairs clearance of bacteria from wounds. Blood. 97:297-304. 